\title{
artigo
}

Pereira, J.S.; Almeida, E.W.S.; Evangelista, C.B.; Dias, C.L.O.; Quadros, J.F.C.; Oliveira, P.A.P.; Dias, O.V.;

Perfil de gestantes atendidas no pré-natal em equipes de estratégia saúde da familia

\section{Perfil de gestantes atendidas no pré-natal em equipes de estratégia saúde da família}

Profile of pregnant women attended prenatal care in family health strategy teams

Perfil de embarazadas mujeres embarazadas en equipos de estrategia de salud familiar

\section{RESUMO}

Objetivo: O presente estudo teve por objetivo analisar o perfil assistencial das gestantes atendidas no pré-natal em equipes de Estratégia Saúde da Familia de um município do Norte de Minas Gerais. Método: Trata-se de um estudo descritivo, transversal, quantitativo e documental, realizado com dados de 6.545 gestantes cadastradas no SISPRENATAL e atendidas em 110 equipes da Estratégias Saúde da Familia do município de Montes Claros, MG, no ano de 2016. Resultados: Os dados mostraram que a maioria das gestantes se declarou parda (55,19\%). A idade predominante estava entre 20 a 34 anos (70,69\%). Em relação a quantidade de consultas de pré natal realizadas, apenas 34,59\% realizaram o número preconizado para a gestação, captadas no primeiro trimestre de gestação (56,52\%) e acompanhadas até a $40^{\mathrm{a}}$ semana de gestação $(29,46 \%)$. Conclusão: 0 estudo possibilitou a análise do perfil assistencial das gestantes atendidas pelas equipes da ESF em um município do Norte de Minas. Observa-se que parte dos indicadores de acompanhamento de pré-natal estão sendo atingidos, o que revela um resultado favorável das equipes de Atenção Primária à Saúde (APS) na assistência pré-natal. Porém percebe-se pontos de fragilidade da assistência prestada que merecem maior reflexão tanto pelas equipes, quanto pelos gestores de forma a aprimorar a qualidade do cuidado às gestantes.

DESCRITORES: Saúde da Mulher; Cuidado Pré-Natal; Estratégia Saúde da Família.

\section{ABSTRACT}

Objective: this study aimed to analyze the profile of pregnant women seen in prenatal outreach teams of the family health strategy of a city in the North of Minas Gerais. Method: this is a descriptive, transversal study, quantitative and documentary, performed with data from pregnant women registered in the SISPRENATAL and 6,545 met in 110 family health Strategies of the city of Montes Claros, MG, in the year 2016. Results: the data showed that most pregnant women pleaded brown ( $55.19 \%$ ). The predominant age was between 20 to 34 years of age (70.69\%). Regarding consultations, of the total of women, only $34.59 \%$ performed the required number for pregnancy, that is, six or more queries; the captured in the first trimester of pregnancy $(56.52 \%)$ and accompanied to the 40 th week of gestation $(29.46 \%)$. Conclusion: the study made it possible to carry out the welfare profile analysis of pregnant women that have been answered in the prenatal care in ESF teams of a municipality of Northern mines. Based on the findings, itcan be observed that part of indicators of antenatal monitoring are being hit with a percentage above $50 \%$, which shows a favorable outcome on the efforts of teams of primary health care (APS). With unfavorable indicators need to reflect, on the part of managers, community and health professionals of the APS for the search of strategies for improving the quality of care to pregnant.

DESCRIPTORS: Women's Health; Prenatal care; Family Health Strategy.

\section{RESUMEN}

Objetivo: Este estudio tuvo como objetivo analizar el perfil de atención de las mujeres embarazadas atendidas en atención prenatal en equipos de Estrategia de Salud Familiar en un municipio en el norte de Minas Gerais. Método: Estudio descriptivo, transversal, cuantitativo y documental, realizado con datos de 6.545 mujeres embarazadas registradas en SISPRENATAL y al que asistieron 110 equipos de Estrategias de Salud Familiar en la ciudad de Montes Claros, MG, en 2016. Resultados: Los datos mostraron que la mayoría de las mujeres embarazadas se declararon marrones $(55,19 \%)$. La edad predominante fue entre 20 y 34 años $(70,69 \%)$. Con respecto al número de consultas prenatales realizadas, solo el 34.59\% realizó el número recomendado para el embarazo, capturado en el primer trimestre del embarazo (56.52\%) y seguido hasta la semana 40 del embarazo (29.46\%). Conclusion: el estudio permitió analizar el perfil de atención de las mujeres embarazadas atendidas por equipos del FSE en un municipio del norte de Minas. Se observa que se están alcanzando parte de los indicadores de monitoreo prenatal, lo que revela un resultado favorable de los equipos de Atención Primaria de Salud (APS) en atención prenatal. Sin embargo, existen debilidades en la atención brindada que merecen una mayor reflexión tanto por parte de los equipos como de los gerentes para mejorar la calidad de la atención a las mujeres embarazadas.

DESCRIPTORES: Salud de la Mujer; Cuidado Prenatal; Estrategia de Salud Familiar.

RECEBIDO EM: 05/12/2019 APROVADO EM: 05/12/2019

\section{Jéssica Santos Pereira}

Bacharel em Enfermagem da Universidade Estadual de Montes Claros. https://orcid.org/0000-0001-5886-1549 


\section{Emerson Willian Santos de Almeida}

Aluno de Mestrado do Programa de Enfermagem Fundamental Escola de Enfermagem de Ribeirão Preto da Universidade de São Paulo - Centro Colaborador da OPAS/OMS para o Desenvolvimento da Pesquisa em Enfermagem. https://orcid.org/0000-0002-6846-021X

\section{Christiane Borges Evangelista}

Professora do curso de graduação em Enfermagem da Universidade Estadual de Montes Claros. Mestre em Ciências pela Universidade Federal de São Paulo - UNIFESP - SP. https://orcid.org/0000-0002-2392-2660

\section{Cristiano Leonardo de Oliveira Dias}

Professor do curso de graduação em Enfermagem da Universidade Estadual de Montes Claros. Doutorando em Enfermagem UNIFESP - SP. https://orcid.org/0000-0002-2750-8416

\section{Joice Fernanda Costa Quadros}

Especialista em Saúde da Família pela Universidade Estadual de Montes Claros. https://orcid.org/0000-0002-7753-951X

\section{Patrícia Alves Paiva de Oliveira}

Mestre pelo Programa de Pós-Graduação Stricto Sensu em Ciências da Saúde da Unimontes. https:/orcid.org/0000-0002-6565-0587

\section{Orlene Veloso Dias}

Professora do curso de graduação em Enfermagem da Universidade Estadual de Montes Claros. Doutora em Ciências pela Universidade Federal de São Paulo - UNIFESP - SP. https://orcid.org/0000-0002-9017-7875

\section{INTRODUÇÃO}

0 pré-natal é o período que antecede o parto, no qual há assistência total às mulheres enquanto gestantes, parturientes e puérperas. O acompanhamento realizado tem como um dos principais objetivos promover o bom desenvolvimento da gravidez e, com isso, o parto saudável. Sendo um momento em que há inserção de ações voltadas à saúde materna e fetal. Essas ações ocorrem durante toda trajetória gestacional para proporcionar atendimentos de qualidade e reduzir a mortalidade materna e perinatal. São desenvolvidas atividades preventivas, educativas e terapêuticas e toda essa assistência é importante para prevenção de agravos, diagnóstico precoce de doenças e tratamentos que se façam necessários nesse período ${ }^{(1,2)}$.

As Unidades Básicas de Saúde (UBS), onde situam as equipes da Estratégias Saúde da Família (ESF) devem ser a porta de entrada das gestantes no Sistema Único de Saúde (SUS), devido à proximidade do contexto familiar e da possibilidade do acompanhamento longitudinal da família. No primeiro, segundo e terceiro trimestres, a assistência prestada se configura como um importante indicador da qualificação dos cuidados realizados à mulher grávida e, sempre que possível, deve envolver a família, em especial o parceiro ${ }^{(3,4)}$.

As equipes da ESF são multiprofissionais, compostas em sua grande maioria por médicos, enfermeiros, técnicos de enfermagem, agentes comunitários de saúde, além de profissionais da saúde bucal e atuam em uma população de 2.000 a 3.500 pessoas. A prestação de serviços à gestante deve ser feita por toda a equipe e vai desde o acompanhamento no domicílio pelo agente comunitário de saúde até as consultas de pré-natal realizadas pelos profissionais médicos e enfermeiros ${ }^{(5,6)}$.

Neste contexto, torna-se importante realizar estudos com o intuito de acrescentar informações a respeito do perfil das gestantes atendidas nas equipes de ESF, podendo trazer subsídios para gestão do cuidado de saúde dessa clientela, contribuindo para a melhoria da qualidade da assistência e diminuindo os riscos de complicações advindas desse período.

Assim, o presente estudo objetivou analisar o perfil assistencial das gestantes atendidas no pré-natal em equipes de Estratégia Saúde da Família de um município do Norte de Minas Gerais.

\section{METODOLOGIA}

Trata-se de um estudo descritivo, transversal, quantitativo e documental realizado com 6.545 gestantes cadastradas nas equipes de ESF, no município de Montes Claros - MG no ano de 2016. O estudo foi feito com os dados obtidos na Secretaria Municipal de Saúde de Montes Claros, através do relatório pré-natal de acompanhamento de gestantes. Esse relatório é gerado pelo software do sistema de informação do SISPRENATAL, que faz parte do Programa de Humanização no Pré-Natal e Nascimento (PHPN) do SUS. A população estudada foi de mulheres que estiveram grávidas no ano de 2016, cadastradas nas 110 equipes de ESF do município. A pesquisa foi censitária, o que justifica não haver uma amostragem, e todo o universo foi trabalhado com o intuito de atingir o objetivo proposto.

$\mathrm{O}$ instrumento de coleta de dados foi elaborado com base nas informações contidas no relatório do SISPRENATAL, segundo as variáveis: total de gestantes cadastradas; raça; idade; consultas; acompanhadas com idade gestacional até 20 semanas que realizaram exames; captadas até a $20^{a}$ semana 
de gestação e acompanhadas até a 40a semana de gestação.

Após a coleta, os dados foram processados e analisados por meio do software Microsoft Office Excel 2010.

Em conformidade com os preceitos da Resolução n. ${ }^{\circ}$ 466, de 12 de dezembro de 2012 do Conselho Nacional de Saúde (CNS), que regulamenta as pesquisas envolvendo seres humanos, este estudo teve seu projeto submetido e aprovado pelo Comitê de Ética em Pesquisa da Universidade Estadual de Montes Claros, sob o parecer n. ${ }^{\circ}$ 2.073.231. Anterior a este, foram assinados o Termo de Concordância da Instituição para Participação em Pesquisa e o Termo de Responsabilidade para Acesso, Manipulação, Coleta e Uso das Informações de Sigilo Profissional para fins Científicos.

\section{RESULTADOS E DISCUSSÃO}

Observou-se que das 6.545 gestantes cadastradas no SISPRENATAL em equipes da ESF, a maioria 3.612 $(55,19 \%)$ era parda (Gráfico 1$)$. Dado semelhante ao encontrado em um estudo realizado em Tocantins com gestantes de UBS, onde um pouco mais da metade das gestantes também se declarava parda $-53,4 \%^{(7)}$. No Brasil, cerca de $50 \%$ da população tem a cor negra,

Gráfico 1. Perfil das gestantes cadastradas no SISPRENATAL quanto à raça/cor. Montes Claros, MG, Brasil, 2016.

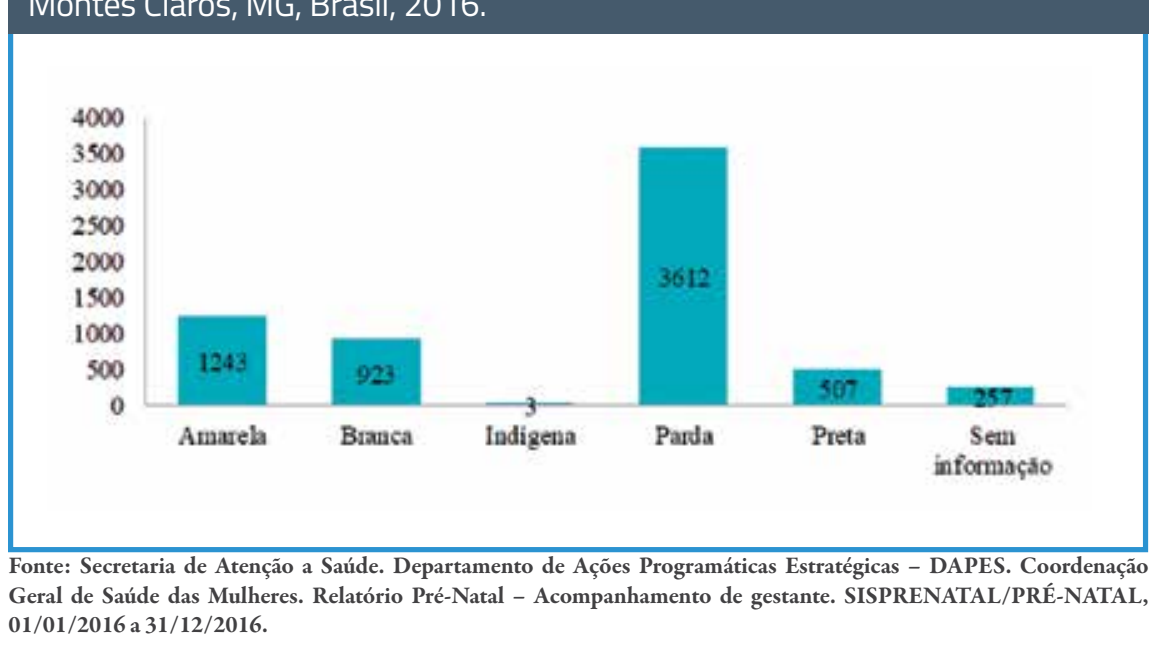

Fonte: Secretaria de Atenção a Saúde. Departamento de Açóes Programáticas Estratégicas - DAPES. Coordenação Geral de Saúde das Mulheres. Relatório Pré-Natal - Acompanhamento de gestante. SISPRENATAL/PRÉ-NATAL, $01 / 01 / 2016$ a $31 / 12 / 2016$.

sendo esta autodeclarada afrodescendente (negros ou pardos), o que justifica o predomínio de gestantes nesse público $^{(8)}$. Nesse estudo houve separação das raças/cores negras e pardas.

Em relação à raça negra, foram encontradas 507 (7,75\%) mulheres (Gráfico 1). Na gestação, a mulher negra possui uma maior predisposição biológica a algumas morbidades, como diabetes mellitus tipo II, pré-eclâmpsia ou eclampsia no parto e diabetes gestacional, além de anemia falciforme, responsável por uma série de complicaçôes na gravidez, como descolamento prematuro de placenta, toxemia grave, natimorto e parto prematuro, requerendo maior atenção dos profissionais da saúde nesse período ${ }^{(7-9)}$.

Outro ponto que merece ser destacado é que estudos demonstram que gestantes de raça parda ou negra, além da predisposição genética para determinadas doenças, enfrentam também uma série de problemas no decorrer da gestação, influenciados por determinantes sociais da saúde. Nesse sentido, a Política de Atenção Integral à Saúde da População Negra busca medidas que promovam a equidade a esse público ${ }^{(10)}$. Além disso, as mulheres negras e pardas possuem menor adesão ao serviço de saúde, o que se torna um agravante considerável para a qualidade de vida materna e fetal, considerando que dificulta a intervenção diagnóstica precoce a algumas doenças ${ }^{(8)}$.

Ainda sobre a variável raça/cor, percebeu-se que $18,99 \%$ das mulheres se autodeclarou raça/cor amarela, que corresponde à origem oriental, raça/ cor pouco prevalente na população brasileira. Encontrou-se 14,10\% mulheres brancas e apenas três indígenas. O percentual de $3,93 \%$ correspondeu a ausência dessa variável, fato preocupante, visto a importância da raça/cor para a gestação, denotando falha no preenchimento do sistema (Gráfico 1).

A idade inferior a 15 anos e acima dos 35 anos é considerada fator de risco pelo Ministério da Saúde. Nesse estudo, observou-se que a maioria das mulheres estava entre a faixa etária requerida como a melhor para gestação, ou seja, 20 a 34 anos de idade com 70,69\% (Gráfico 2). Com predomínio das faixas entre 20 a 24 anos $(25,18 \%)$ e 25 a 29 anos (25,91\%). A gestação com a idade recomendada pelo Ministério da Saúde visa o menor número de intercorrências no período ${ }^{(9)}$.

Segundo a Organização Mundial da Saúde (OMS), a fase da adolescência compreende dos 10 aos 19 anos de idade. Quanto a esse público, observou-se que o maior número de gestantes adolescentes estava entre a faixa etária de 15 a 19 anos (13,08\%), seguida de uma menor quantidade na faixa etária entre 10 a 14 anos de idade $(0,31 \%)$, onde se tem maior risco gestacional (Gráfico 2). Número bem reduzido em relação à década de 90 , na qual quase $26 \%$ dos partos no Brasil era de adolescentes ${ }^{(12)}$.

Ressalta-se que a gravidez na adolescência, em comparação com a gestação na idade recomendada, é de maior risco, uma vez que é verificada maior ocorrência de complicações na gravidez e durante o parto. Complicações como, anemia, maior número de doença hipertensiva, menor ganho de peso, parto prematuro, anoxia, baixo peso no nascimento, entre outras ${ }^{(12)}$. Não foi encontrado registro de gravidez 
em idade inferior a 10 anos. Os dados encontrados nesse estudo em relação à quantidade de adolescentes grávidas revelam o sucesso das ações de prevenção da gravidez na adolescência pela atenção primária.

Observou-se que $15,92 \%$ das mulheres deste estudo teve a gestação acima dos 34 anos (Gráfico 2). As incidências indicam que a gestação tardia, ou seja, em mulheres acima dessa idade, têm aumentado cada vez mais ao longo dos anos e diferentes fatores podem ter contribuído com esse aumento, onde se destaca a inserção da mulher ao mercado de trabalho, melhor nível socioeconômico e educacional ${ }^{(14)}$.

A gravidez tardia pode trazer à mulher e ao feto diferentes complicações, como: maior ocorrência de gravidez ectópica, parto prematuro, baixo peso no nascimento, baixa vitalidade do recém-nascido, abortamentos espontâneos e maior mortalidade perinatal ${ }^{(15)}$. Essas mulheres ainda podem apresentar maior risco para diabetes e doenças hipertensivas ${ }^{(12)}$. A ocorrência da hipertensão arterial se relaciona à alteração nas vascularizações, decorrente da idade. Em mulheres acima de 35 anos, pode ser encontradas com maior frequência hemorragias periparto, além de maior número de partos operatórios, parto prematuro, amniorrexe prematura e placenta prévia ${ }^{(14)}$. A gestação, nesse quesito, requer uma atenção ainda mais acentuada dos profissionais da saúde, ainda que existam gestantes com a mesma faixa etária e sem necessidade de intervenções específicas.

Aliada à boa qualidade da assistência prestada à mulher enquanto gestante, o estudo trouxe alguns dos indicadores da qualidade da assistência ao pré-natal (proporção de gestantes cadastradas no

Gráfico 2. Perfil das gestantes cadastradas no SISPRENATAL quanto à idade. Montes Claros, MG, Brasil, 2016.

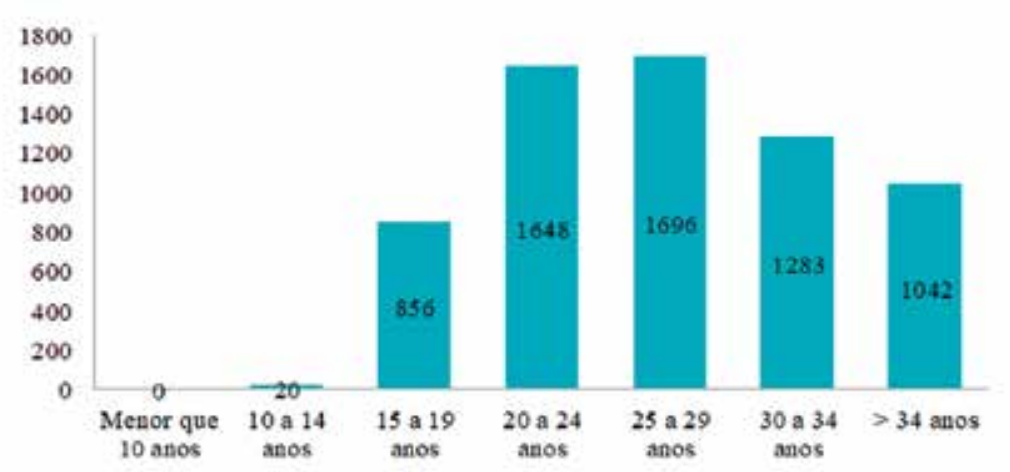

Fonte: Secretaria de Atenção a Saúde. Departamento de Açóes Programáticas Estratégicas - DAPES. Coordenação Geral de Saúde das Mulheres. Relatório Pré-Natal - Acompanhamento de gestante. SISPRENATAL/PRÉ-NATAL, $01 / 01 / 2016$ a $31 / 12 / 2016$.

Tabela 1. Perfil das gestantes cadastradas no SISPRENATAL quanto à consulta. Montes Claros, MG, Brasil, 2016.

$$
\text { VARIÁVEIS }
$$$$
\text { N (6.545) }
$$

Consultas

6 ou mais consultas

Acompanhadas com IG até 20 semanas que realizaram exames

Fonte: Secretaria de Atenção a Saúde. Departamento de Ações Programáticas Estratégicas - DAPES. Coordenação Geral de Saúde das Mulheres. Relatório Pré-Natal - Acompanhamento de gestante. SISPRENATAL/PRÉ-NATAL, $01 / 01 / 2016$ a $31 / 12 / 2016$. pré-natal; proporção de gestantes com seis ou mais consultas de pré-natal; proporção de gestantes acompanhadas no pré-natal que realizou exames de $\mathrm{Hb}$, Hct, Glicemia, EAS, VDRL e HIV até a $20^{a}$ semana de gestação; proporção de gestantes com captação precoce no pré-natal), além da proporção de gestantes acompanhadas até a $40^{\text {a }}$ semana de gestação, com base no Programa de Humanização no pré-natal e Nascimento e pautados no SISPRENATAL. Esses indicadores tornam possível a Rede Cegonha proporcionar a avaliação da qualidade da assistência. A Rede Cegonha foi lançada em 2011 pelo Ministério da Saúde e normatizada pela Portaria n. ${ }^{\circ} 1.459$, cujo objetivo é prestar uma boa qualidade na assistência ao pré-natal, parto, puerpério e a criança até 24 meses e avaliar os indicadores. Esses indicadores auxiliam os gestores na promoção da saúde, pois propiciam conhecer e identificar a realidade da condição de saúde dessa população. No pré-natal é ainda mais importante, por proporcionar um apoio necessário à gestação ${ }^{(16,17)}$.

Com o intuito de reorganizar as ações realizadas no âmbito da gestação, os Sistemas de Informação em Saúde (SIS) desempenham importante papel na função administrativa dos atendimentos. O SISPRENATAL é um dos sistemas de suporte no gerenciamento dessas ações desenvolvidas para as gestantes inseridas no PHPN do SUS. Neste sistema, é possível realizar o acompanhamento e monitoramento da gestação desde o primeiro atendimento ao puerpério, pois elenca os indicadores da assistência destinada à gestação.

Em relação à quantidade de consultas, percebeu-se que menos da metade, 2.264 (34,59\%), tinha o número de 6 ou mais consultas de pré-natal, conforme recomendado pelo Ministério da Saúde (Tabela 1). Número baixo comparado com um estudo realizado em duas maternidades existentes do Município de Rio Grande, extremo sul do Brasil, onde cerca de mais de $60 \%$ das mulheres tinha seis ou mais consultas. No tocante a esse indicador, os dados deste estudo não especificam se elas foram realizadas por profissionais médi- 
cos, enfermeiros ou algum outro, embora a consulta de pré-natal possa ser feita pelos dois profissionais ${ }^{(18,19)}$.

O principal objetivo do PHPN é a redução das taxas de morbimortalidade, além de buscar melhorar o acesso ao pré-natal por meio da humanização. Tal programa fornece informações para atender às necessidades específicas da gestante, feto e a mulher após o parto, buscando assegurar a melhoria na qualidade e acesso do pré-natal, parto e puerpério. No PHPN é incluído o número mínimo de consultas no pré-natal. No primeiro trimestre, faz-se necessário uma consulta, duas no segundo trimestre e três no terceiro trimestre, totalizando seis consultas. Até a $28^{a}$ semana, as consultas são realizadas mensalmente; a partir da $28^{\mathrm{a}}$ quinzenalmente e da $36^{\mathrm{a}}$ até a $41^{\text {a }}$ semana, as consultas são semanalmente. Além de uma consulta no período puerperal até 42 dias após o parto ${ }^{(9,20)}$.

No último trimestre, faz-se necessário um número maior de consultas devido, principalmente, à aproximação do parto e aumento da probabilidade de intercorrências com o avançar da gestação. Mesmo assim, $1.928(29,46 \%)$ do total das mulheres foram acompanhadas até a $40^{\mathrm{a}}$ semana de gestação (Tabela 1). Esse quantitativo não indica os reais motivos do não acompanhamento até esse período, ou seja, pode ser devido também ao parto anterior. $\mathrm{O}$ último trimestre requer uma atenção redobrada dos profissionais da saúde com um número superior de consultas, que pode ser até acima do recomendado de três ${ }^{(9,20)}$.

Em se tratando das consultas realizadas, a primeira geralmente é feita pelo profissional enfermeiro, de forma detalhada, preconizando a realização de anamnese, exame físico e orientações à gestante. As consultas subsequentes são divididas entre o médico e o enfermeiro. As consultas podem ser realizadas tanto nas UBS quanto em domicílio. A diminuição do número de consultas pode estar relacionada à dificuldade de acesso aos serviços de saúde, o que pode ocasionar o nascimento pré-termo, assim como o baixo ganho de peso da mulher no período gestacional, dentre outras consequências ${ }^{(9,21)}$.
No âmbito

do SUS e por

meio da Rede

Cegonha, a

mulher com

suspeita de

gravidez é

captada na

própria UBS e

submetida ao

Teste Rápido de

Gravidez (TIG)

para confirmação

da hipótese

e início dos

\section{atendimentos no}

pré-natal.

Em relação às gestantes captadas até a $12^{a}$ semana de gestação, o número ultrapassa $50 \%$ das cadastradas - 56,52\% (Tabela 1). No Programa Nacional de Melhoria do Acesso e da Qualidade da Atenção Básica (PMAQ), existe um indicador que avalia a proporção de gestantes que iniciaram o pré-natal no $1^{\circ}$ trimestre, de acor- do com os dados retirados do Sistema de Informação da Atenção Básica (SIAB) de 2010 , cerca de $79 \%$ das mulheres acompanhadas foram captadas no $1^{\circ}$ trimestre da gestação. Tal indicador traz à tona $o$ percentual de mulheres que tiveram início precoce ao pré-natal. Quando inseridas desde o início da gestação ao programa, as mulheres são acompanhadas e diagnosticadas precocemente, caso tenham alguma alteração nesse período ${ }^{(22)}$.

No âmbito do SUS e por meio da Rede Cegonha, a mulher com suspeita de gravidez é captada na própria UBS e submetida ao Teste Rápido de Gravidez (TIG) para confirmação da hipótese e início dos atendimentos no pré-natal. No caso de atraso menstrual de 15 dias ou mais, o médico ou o enfermeiro pode solicitar o TIG e inserir a gestante no programa, ou seja, concluindo sua captação, com o início do acompanhamento no SISPRENATAL ${ }^{(9)}$.

No que diz respeito às mulheres acompanhadas com idade gestacional (IG) até 20 semanas que realizaram exames, refere-se à contabilização daquelas que realizaram exames da lista de indicadores do SISPRENATAL, que são eles: Hemograma, VDRL, Glicemia, Urocultura e HIV, ainda que haja outros exames para o mesmo período. Percebe-se que houve um número bem reduzido de adesão aos exames preconizados $(6,60 \%)$, indicando uma baixa cobertura quando comparados aos padrões indicados pelo Ministério da Saúde (Tabela 1). Os exames na gestação são importantes por proporcionar o acompanhamento e possível intervenção à mulher, uma vez que possibilitam o diagnóstico precoce de doenças que podem acarretar complicações durante a gestação. A boa qualidade do acompanhamento se relaciona à realização dos exames preconizados, uma vez que os mesmos têm o mais alto grau de indicação, devendo ser realizados, a grande maioria, na primeira consulta, por se tratar do primeiro contato da gestante ao serviço de saúde ${ }^{(9)}$.

\section{CONCLUSÃO}

O estudo possibilitou realizar a análise 
do perfil assistencial das gestantes atendidas no pré-natal em equipes de ESF de um município de porte médio do Norte de Minas Gerais. Com base nos achados, pode-se observar que houve baixa adesão das mulheres ao número mínimo de consultas de pré-natal exigido pelo Ministério da Saúde. A maioria das mulheres, pouco mais da metade, foi captada até a $12^{\mathrm{a}} \mathrm{se}-$ mana de gravidez. Observou-se ainda a predominância de mulheres entre as faixas etárias de 20 a 29 anos, requerido como melhor período para gestação e baixo percentual de gravidez na adolescência $(13,39 \%)$. Notou-se maior número da raça/cor parda. Houve baixa cobertura dos exames até a $20^{a}$ semana e menos da metade das mulheres foram acompanhadas até o final da gestação.

Diante desses resultados, nota-se que as equipes da APS do município estudado têm obtido resultados favoráveis na captação precoce das gestantes e baixo índice de gravidez na adolescência. No que tange ao número de consultas de pré-natal e cobertura de exames realizados, percebe-se pontos de fragilidade na assistência prestada que merecem maior reflexão tanto pelas equipes, quanto pelos gestores, de forma a aprimorar a qualidade do cuidado às gestantes. "

\section{REFERÊNCIAS}

1. Barreto CN, Ressel LB, Santos CC, Wilhelm LA, Silva SC, Alves CN. Atenção Pré-Natal na voz das gestantes. Rev Enferm UFPE on-line. 2013;7(5):4354-63.

2. Ministério da Saúde (BR). Portal da Saúde. Departamento de Atenção Básica. e-SUS Atenção Básica. Brasília; 2012.

3. Cunha MA, Mamede MV, Dotto LMG, Mamede MV. Assistência Pré-Natal: competências essenciais desempenhadas por enfermeiros. Esc Anna Nery Rev Enferm. 2009; 13 (1): 00-00.

4. Andrade MV, Noronha K, Barbosa ACQ, Rocha TAH, Silva NC, Calazans JA, et al. A equidade na cobertura da Estratégia Saúde da Família em Minas Gerais, Brasil. Cad Saúde Pública. 2015;31(6):1175-1187.

5. Ministério da Saúde (BR). Portaria n. ${ }^{\circ} 2.436$, de 21 de setembro de 2017. Aprova a Política Nacional de Atenção Básica, estabelecendo a revisão de diretrizes para a organização da Atenção Básica, no âmbito do Sistema Único de Saúde (SUS). Setembro de 2017.

6. Rodrigues EM, Nascimento RG, Araújo A. Protocolo na assistência pré-natal: ações, facilidades e dificuldades dos enfermeiros da Estratégia de Saúde da Família. Rev Esc Enferm USP. 2011;45(5):1041-7

7. Silva MG, Gontijo EE L, Ferreira DS, Carvalho FS, Castro AM. O perfil epidemiológico de gestantes atendidas nas unidades básicas de saúde de Gurupi, Tocantins. Universitas: Ciências da Saúde. 2015;13(2):93-102.

8. Cunha EMGP. 0 recorte racial no estudo das desigualdades em saúde. São Paulo em Perspectiva. 2008;22(1):79-91.

9. Ministério da Saúde (BR). Secretaria de Atenção à Saúde. Departamento de Atenção Básica. Atenção ao pré-natal de baixo risco / Ministério da Saúde. Secretaria de Atenção à Saúde. Cad 32. Departamento de Atenção Básica. - Brasília: Editora do Ministério da Saúde, 2012.

10. Pacheco VC, Silva JC, Mariussi AP, Lima MR, Silva TR. As influências da raça/cor nos desfechos obstétricos e neonatais desfavoráveis. Saúde debate. 2018;42(116).

11. Ministério da Saúde (BR). Departamento de Informática do SUS. Informações de saúde (TABNET). Estatísticas Vitais [Internet]. Brasília: Ministério da Saúde [cited 2018 Jan. 9]. Available from: http://www2.datasus.gov.br/ DATASUS/index.php?area $=0205$.

12. Ximenes FMA, Oliveira MCR. A influência da idade materna sobre as condições perinatais. RBPS. 2004;17(2):56-60.

13. Silva CR, Lopes RE. Adolescência e juventude: entre conceitos e políticas públicas. Cadernos de Terapia Ocupacional da UFSCar. 2009: 87-106.

14. Gonçalves ZR, Monteiro DLM. Complicações maternas em gestantes com idade avançada. FEMINA. 2012;40(5).

15. Aldrighi JD, Wall ML, Souza SRRK, Cancela FZV. As experiências das mulheres na gestação em idade materna avançada: revisão integrativa. Rev Esc Enferm USP. 2016;50(3):512-21.

16. Lima KWS, Antunes JLF, Silva ZP. Percepção dos gestores sobre o uso de indicadores nos serviços de saúde. Saúde Soc. São Paulo. 2015;24(1):61-71.

17. Cavalcanti PCS, Gurgel Junior GD, Vasconcelos ALR, Guerrero AVP. Um modelo lógico da Rede Cegonha. Physis. 2013;23(4).

18. Barbosa JTC, Vettori TNB, Saldanha BL, Rocha RM, Braga ALS, Andrade M. Sisprenatal como ferramenta facilitadora da assistência à gestante: revisão integrativa da literatura. Revista de Atenção à Saúde. 2014;12(42):42-7.

19. César JA, Mendonza-Sassi RA, Gonzalez-Chica DA, Mano PS, GoulartFilha SM. Características sociodemográficas e de assistência à gestação e ao parto no extremo sul do Brasil. Cad Saude Publica. 2011;27(5):985-94.

20. Silva KMC, Silva KMC. Caracterização dos perfis das gestantes atendidas na UBS no município de Campos Gerais - MG. Campos Gerais. Monografia [Bacharelado em Enfermagem] Faculdade de Ciências e Tecnologia de Campos Gerais; 2010.

21. Souza NA, Queiroz LLC, Queiroz RCCS, Ribeiro TSF, Fonseca MSS. Perfil epidemiológico das gestantes atendidas na consulta de pré-natal de uma Unidade Básica de Saúde em São Luís-MA. Rev Ciênc Saúde. 2013;15(1):28-8.

22. Ministério da Saúde (BR). Programa Nacional de Melhoria do Acesso e da Qualidade da Atenção Básica (PMAQ). Manual instrutivo - Anexo Ficha de Qualificação dos Indicadores. Brasília - DF, 2012. 